\title{
A Prediction Model for Thin-walled Duct Structure based with RBF Neural Network
}

\author{
Jianbing Wang ${ }^{1, a}$, Wen Zhang ${ }^{2, b}$, Yueqiang Zhang ${ }^{1, c}$ and Yingsheng Huang ${ }^{2, d}$ \\ ${ }^{1}$ China North Vehicle Research Institute, Beijing 100072, China; \\ ${ }^{2}$ School of Mechanical Engineering, Dalian University of Technology, Dalian 116024, China.

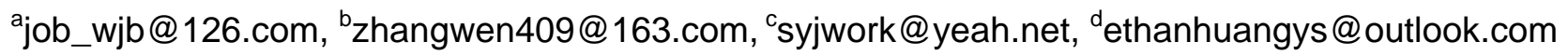

Keywords: RBF neural network, thin-walled duct structure design, forecasting design, ABAQUS.

\begin{abstract}
Component structure design is based on the finite element analysis technology, and the structure optimization is obtained by the iterative process of the boundary. However, this method is time-consuming and expensive to deal with the numerical simulation or complex engineering problems. In this paper, we combined the technology of experimental design and RBF neural network model technology, established a high accuracy analysis and prediction model of the structural shape by using a small number of sample points. We instituted the intrinsic part model and found the structural parameters within the constraints in the surrogate model. Consequently, we predicted the optimize structure of the part. Firstly, this paper described the design idea and theoretical basis of the method, and then illustrated the application process of the method with the example of the structure optimization of thin walled duct. The experimental results showed that this method can improve the computational efficiency and the design quality of the structure.
\end{abstract}

\section{Introduction}

Part structural optimization is to reduce the stress concentration and improve the characteristics of the structure by changing the structural parameters of the regional boundaries, and to meet the requirements of certain physical quantities on the boundary. At present, the high precision numerical analysis method is widely used, and combining the high precision numerical analysis with the optimization method organically, launching a comprehensive search and optimization to the design space, has become an important developmental direction of modern design optimization method. Based on ANSYS optimization platform, Lu Shan et al. [1] established a three-dimensional parametric model of the whole leaf disc and the structural optimization design of the mathematical model, and they completed the optimum design of the whole leaf disc. Mai Yunfei et al. [2] used Workbench ANSYS to analyze the modal analysis and stiffness analysis of the panel of the car dashboard, and finally conclude a conclusion that the honeycomb panel is the optimal structure.

In essence, the optimal design is a process of repeated iteration, and the global search ability of traditional optimization algorithm (such as genetic algorithm, simulated annealing algorithm) often require thousands of times call analysis model. In order to improve the optimization efficiency, it is widely used in parts optimization design optimization based agent model. Y. Mack et al. [3] combined with computational fluid dynamics, using response surface method to establish proxy model to achieve the body shape optimization. Xitao Lai et al. [4] can not only get a good optimization results Kriging model based on genetic algorithm combined with centrifugal impeller structure optimization design method than the direct search optimization using genetic algorithms to reduce a lot of computing time and improve design efficiency. Pan et al. [5] used support vector machine agent modeling technology to achieve a significant reduction in the weight of the car $\mathrm{B}$ column. Kim et al. [6] proposed a hybrid multi-objective evolutionary algorithm (mixed MOEA), response surface approximation model is introduced and applied to optimize the design of axial fan blades.

Firstly, the paper describes the structure design method of the design technique and RBF neural network model. Then, the finite element analysis model is established by using ABAQUS software. 
Finally, the RBF neural network model is constructed and the optimal structure is obtained by using the particle swarm optimization algorithm. The correctness and efficiency of the proposed design method are verified by the optimization results.

\section{RBF neural network model and structure prediction design method}

Today, neural network has been widely used in function approximation, pattern recognition, image processing and computer vision, signal processing, time series, medical control, expert system, power system, military system, financial system, artificial intelligence and optimization. The advantages of neural network model include: strong approximation of complex nonlinear function; without mathematical assumptions, and has the characteristics of black box; fast learning speed, with excellent generalization ability, strong fault tolerance, even if samples contain noise input, does not affect the overall performance of the network.

Three layers of feedforward neural networks are shown in Figure 1, The unit layer of the received input signal is called an input layer, the output signal is called the output layer, and the unit layer which is not directly connected with the input and output is called the intermediate layer or the hidden layer. From the input layer to the hidden layer is a fixed nonlinear transformation, the input vector is mapped directly to a new space. The mapping from the hidden layer to output layer space is linear, and the output layer is linear weighted in the new linear space.

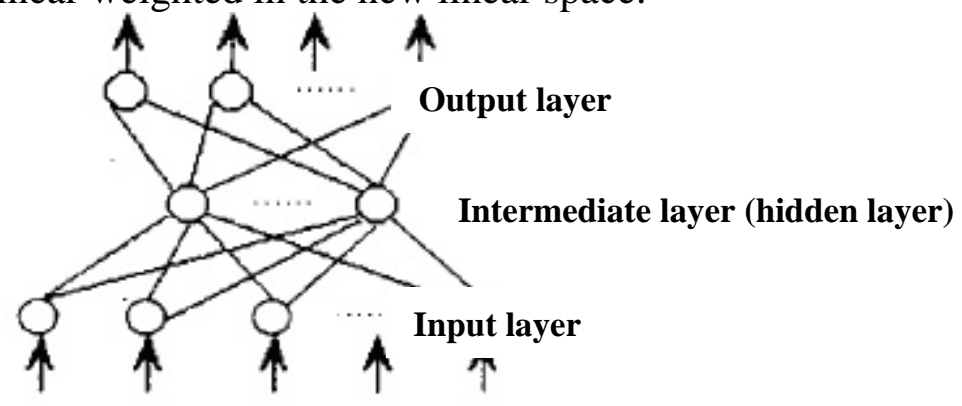

Fig.1 Three layer forward neural network

Radial Basis RBF network: the Euclidean distance between the target point and the sample point for the independent variable, which assumes $x_{1}, \ldots, x_{N} \in \Omega \subset R^{N}$ represents a set of input vectors, $g_{i} \equiv g\left(\left\|x-x_{j},\right\|^{c}\right) \in R, j=1 \ldots N$ is an odd function. Where $\left\|x-x_{j},\right\|$ is the Euclidean distance: $\left(x-x_{j}\right)^{T}\left(x-x_{j}\right)$, and $0.2 \leq c \leq 3$. We used $R^{2}$ as evaluation method as follows:

$$
R^{2}=1-\frac{\sum_{\mathrm{i}=1}^{N}\left(\hat{f}_{i}-\bar{f}_{i}\right)^{2}}{\sum_{\mathrm{i}=1}^{N}\left(f_{i}-\bar{f}_{i}\right)^{2}}
$$

where $f_{i}$ is the response value, $\bar{f}_{i}$ is the average value of $f_{i}, \hat{f}_{i}$ is the predicted value, $N$ is the number of error analysis points.

In this paper, the design method based on the structure prediction RBF neural network. Firstly, we used ABAQUS to construct the finite element analysis model; then used the optimal Latin hypercube design method to construct the design sample point; used the ABAQUS finite element analysis model to obtain the sample response data; finally, we used the RBF method to construct the approximate surrogate model, and use the particle swarm optimization algorithm to optimize the structure of the part. Design flow structure prediction method based on RBF neural network shown in Figure 2. 


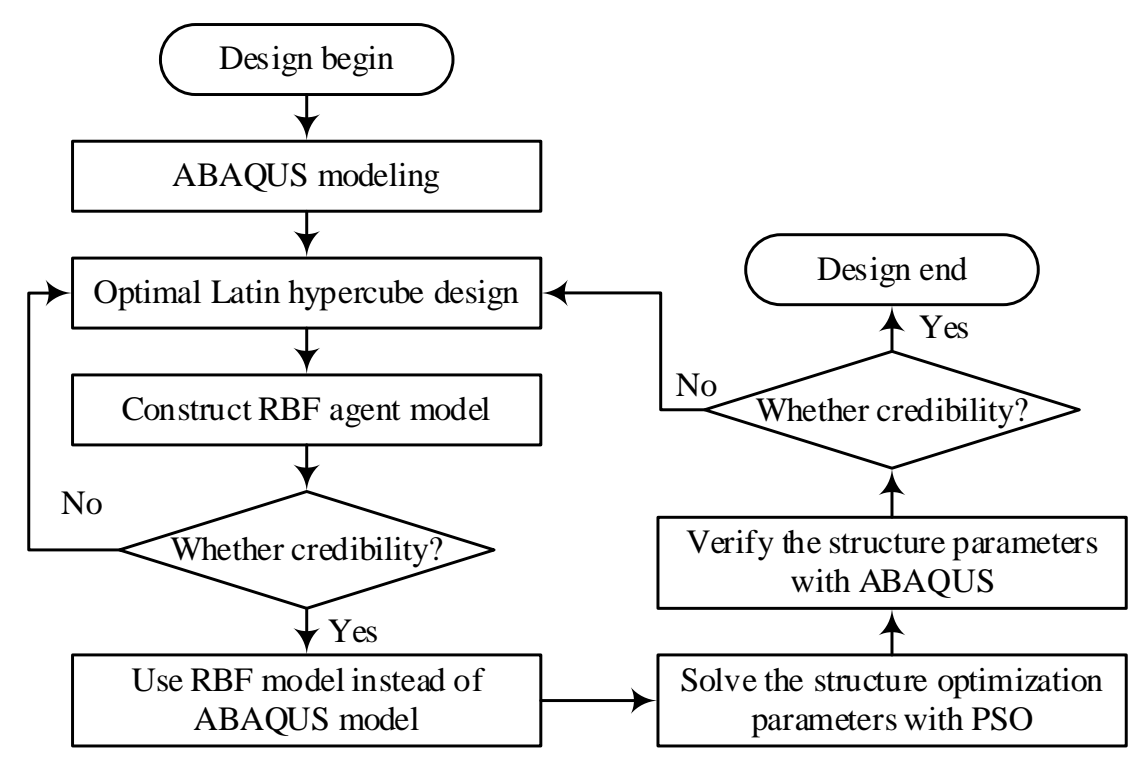

Fig. 2 Structural design method based on RBF neural network

\section{Thin-walled duct Case analysis}

As shown in Figure 3, the thin wall duct structure, which is installed in the aircraft engine and the working environment is bad, the stress situation is very complicated. Expect to get smaller quality, and improve the stress concentration, the maximum structure of fatigue life. A finite element analysis model is established in ABAQUS, and the load is applied (in Table 1), Select the tube thickness (Thickness Duct), the front flange thickness (Thickness Fwd.Flange), rear flange thickness (Thickness Aft flange) as a design variable. Maximum stress (Max.stress) and the safety factor (FOS) as constraints, duct quality (MASS) as a design goal, in Table 2.

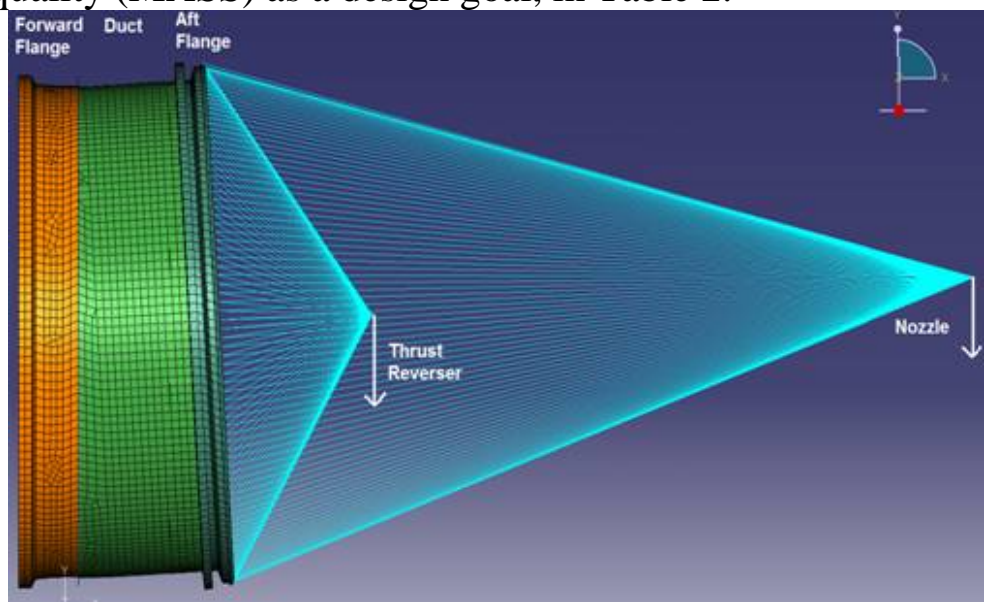

Fig.3 finite element analysis model of thin walled tube

Table 1 loads and boundary conditions

\begin{tabular}{lr}
\hline Loads and Boundary Conditions & \multicolumn{1}{c}{ value } \\
\hline Emergency Landing Acceleration X (g) & 9.00 \\
Emergency Landing Acceleration Y (g) & -3.00 \\
Emergency Landing Acceleration Z (g) & 6.00 \\
Engine Pressure (PSI) & 32.0000 \\
Preliminary Nozzle Weight (lbs) & 250.0000 \\
Preliminary Thrust Reverser Weight (lbs) & 270.0000 \\
\hline
\end{tabular}


Table 2 constraint conditions and objective functions

\begin{tabular}{lr}
\hline Constrain and Objective & \multicolumn{1}{c}{ value } \\
\hline Max.stress (lbs) & $<34025$ \\
FOS & $>2$ \\
MASS (lbs) & minimise \\
\hline
\end{tabular}

We used the optimal Latin hypercube sampling design to obtain 122 sample points in the measured response of ABAQUS, then set up a proxy model RBF neural network. RBF agent model of maximum stress and quality were 0.99862 and 0.99958 , with a high degree of confidence. RBF fitting response curve shown in Figure 4.
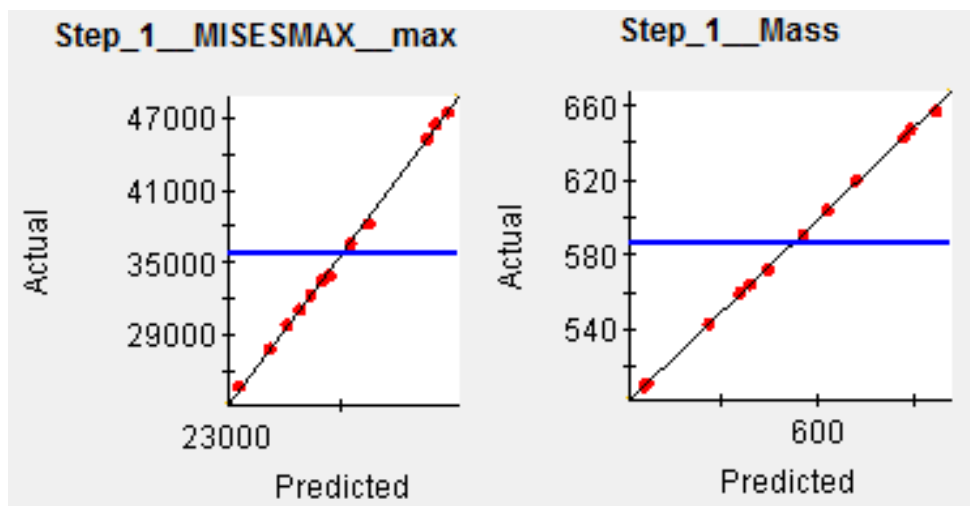

Fig.4 RBF response curve

We used PSO to optimize the RBF model, and the structural parameters corresponding to the minimum mass of the model are solved. The optimized parameters are as follows: the maximum number of iterations is 100 , the number of particles is 20 , the inertia weight is 0.9 , the global increment is 0.9 , the particle increment is 0.9 , the maximum speed is 0.1 . The design objectives and constraints of the optimization curve are shown in figure 5 . Get the optimized structure parameter value back to the ABAQUS model, get the optimization results in table 3 .
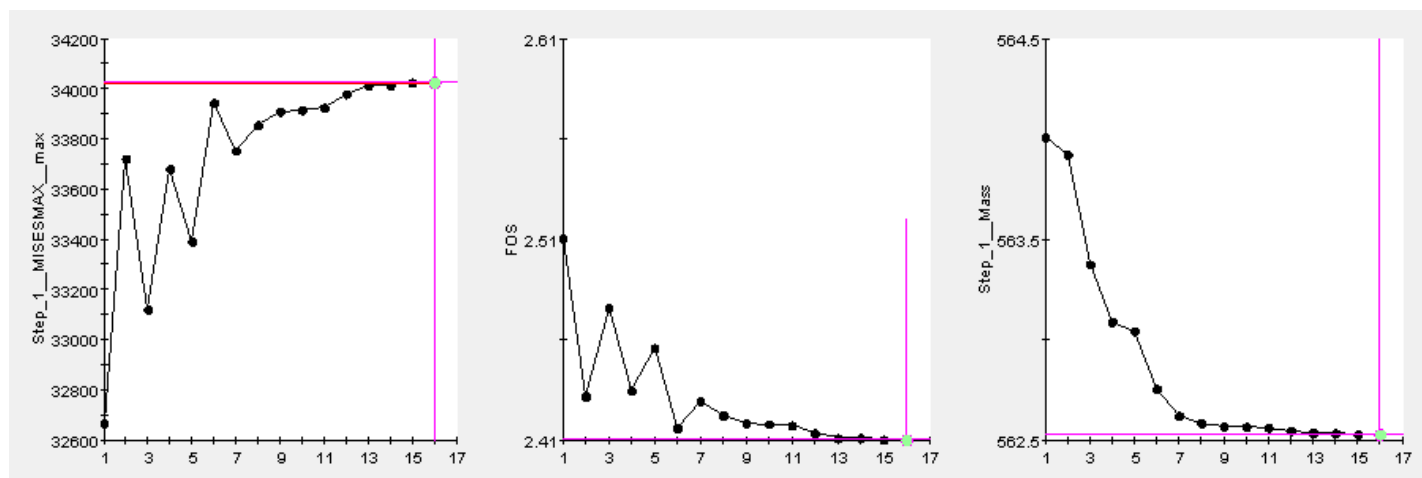

Fig.5 PSO optimization curve

Table 3 optimization results

\begin{tabular}{lccc}
\hline \multicolumn{1}{c}{ Parameters } & Initial model & Optimized model & Optimized ratio \\
\hline Thickness Duct (in) & 0.063 & 0.0504 & \\
Thickness Fwd.Flange (in) & 0.15 & 0.12 & \\
Thickness Aft Flange (in) & 0.15 & 0.137 & \\
Max.stress (lbs) & 33787.1 & 24036.6 & $28.8 \%$ \\
FOS & 2.427 & 2.001 & \\
MASS (lbs) & 567.7 & 560.2 & $1.3 \%$ \\
\hline
\end{tabular}

According to the optimization results, the maximum stress of the structure is reduced from 33787.1 (lbs) to 24036.6 (lbs), the optimal rate is $28.8 \%$ (lbs), and the quality is reduced to 560.2 (lbs), the optimized rate is $1.3 \%$, which achieves the purpose of reducing the quality. 


\section{Summary}

In this paper, the experimental design technique and RBF neural network surrogate model are used to construct the high accuracy analysis and prediction model, which is close to the actual model, and to replace the original part model, which greatly reduces the computational quantity of ABAQUS. And it is convenient to control the particle swarm algorithm to find the optimal design point in the design area, which is in line with the constraint conditions, and obtain the structural optimization parameters. The optimization results of thin walled duct structure showed that this method greatly improves the efficiency and quality of design optimization.

\section{Acknowledgement}

This work is supported by National Natural Science Foundation of China (No. 61304206), and the Program of Liaoning Science and Research (No. L2013460).

\section{Reference}

[1]LU Shan, LU Feng-jie. Structure optimization design for blisk based on ANSYS. Journal of Aerospace Power. Vol. 27(2012) No. 6, p. 1218-1220.

[2]MAI Yun-fei, LIU Wei. Optimal Structural Design Based on ANSYS for Automotive Lateral Cover Plate. Mechanical Engineering \& Automation. Vol. 1(2015) No.1, p. 036.

[3]Y. Mack, T. Goel, W. Shyy, R. Haftka, N. Queipo. Multiple surrogates for the shape optimization of bluff body-facilitated mixing. In Proceedings of the 43rd AIAA aerospace sciences meeting and exhibit. Reno, NV, 2005, p. 2005-0333.

[4]LAI Xi-tao, WEN Wei-dong, FENG Da-jun. Structural optimization of a centrifugal impeller based on a Kriging model. Journal of Shenyang Aerospace University. Vol. 31(2014) No. 4, p. $17-22$.

[5]F. Pan, P. Zhu, Y. Zhang. Metamodel-based lightweight design of B-pillar with TWB structure via support vector regression. Computers \& structures. Vol. 88(2010), p. 36-44.

[6]J.-H. Kim, J.-H. Choi, A. Husain, K.-Y. Kim. Performance enhancement of axial fan blade through multi-objective optimization techniques. Journal of mechanical science and technology. Vol. 24(2010), p. 2059-2066. 